\title{
Evidence for Calreticulin Attenuation of Cardiac Hypertrophy Induced by Pressure Overload and Soluble Agonists
}

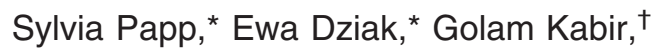 \\ Peter Backx, ${ }^{\dagger}$ Sophie Clement, ${ }^{\ddagger}$ and Michal Opas* \\ From the Department of Laboratory Medicine and Pathobiology," \\ and the Heart and Stroke/Richard Lewar Centre of Excellence, ${ }^{\dagger}$ \\ University of Toronto, Toronto, Ontario, Canada; and \\ Department of Clinical Pathology, ${ }^{\ddagger}$ University of Geneva, \\ Geneva, Switzerland
}

While calreticulin has been shown to be critical for cardiac development, its role in cardiac pathology is unclear. Previous studies have shown the detrimental effects on the heart of sustained germline calreticulin overexpression, yet without calreticulin, the heart does not develop normally. Thus, carefully balanced calreticulin levels are required for the heart to develop and to function properly into adulthood. But what happens to calreticulin levels, and how is this regulated, during cardiac hypertrophy, during which the fetal gene program is reactivated, at least partially? Our working hypothesis was that c-Src, a kinase whose activity we previously found to be correlated with calreticulin expression, was involved with calreticulin in regulating the response to hypertrophic signals. Thus, we subjected adult mice to transverse aortic constriction to induce left ventricular hypertrophy. We found that aortic constriction caused calreticulin levels to increase, whereas those of c-Src fell with longer constriction time. We also examined the ability of embryonic stem cell-derived cardiomyocytes to respond to soluble hypertrophic agonists. Endothelin-1 treatment caused a significantly greater cell area increase of calreticulin-null cardiomyocytes, which had higher c-Src activity, compared with wildtype cells. c-Src inhibition abolished this difference. Greater c-Src activity may explain the efficacy with which calreticulin-null cells are able to induce the hypertrophic program, while cells containing calreticulin may be able to attenuate the hypertrophic response as a result of decreased c-Src activity. Thus, calreticulin may have a protective effect on the heart in the face of cardiac hypertrophy. (Am J Pathol 2010, 176:1113-1121; DOI: 10.2353/ajpath.2010.090392)

Cardiac calreticulin was vaulted into the spotlight by the unexpected discovery that the ablation of calreticulin by homologous recombination causes embryonic lethality at embryonic day 14.5 in mice because of faulty cardiac organogenesis. This was a surprising finding because the levels of calreticulin in the adult heart are very low, and calreticulin has been described as mainly an endoplasmic reticulum-resident protein, with the major $\mathrm{Ca}^{2+}$ binding function of the sarcoplasmic reticulum being attributed to calsequestrin. Furthermore, if high levels of calreticulin are maintained in the heart postnatally, complete heart block and early, postnatal sudden death ensues. ${ }^{1,2}$ The majority of these calreticulin overexpressing mice die within 22 days after birth and none survive past 5 weeks. Thus, both the absence and overexpression of calreticulin in the heart are detrimental.

The hypertrophic response is initiated in the heart in response to a variety of mechanical, hemodynamic, hormonal, and pathological stimuli. ${ }^{3}$ Cardiac muscle mass increases with hypertrophy, and the walls of the chambers, usually the ventricles, thicken because mature cardiomyocytes cannot undergo proliferation. It is believed that in pathological hypertrophy, the cardiomyocytes make an attempt to achieve proliferative capacity by re-expressing the embryonic gene program, including

Supported by grants from Canadian Institutes of Health Research (MPO36384) and from the Heart and Stroke Foundation of Ontario (T 6181).

Accepted for publication October 27, 2009.

S.P. is a recipient of a Canada Graduate Scholarship from the Canadian Institutes of Health Research. M.O. is a member of the Heart and Stroke/ Richard Lewar Centre of Excellence.

Supplemental material for this article can be found on http://ajp. amjpathol.org.

Address reprint requests to Michal Opas, Ph.D., Department of Laboratory Medicine and Pathobiology, University of Toronto, 1 King's College Circle Medical Sciences Building, Room 6326, Toronto, Ontario, M5S 1A8 Canada. E-mail: m.opas@utoronto.ca. 
fetal forms of myosin heavy chain ( $\mathrm{MHC}$ ), atrial natriuretic protein, and skeletal $\alpha$-actin genes. ${ }^{4}$

The cardiac hypertrophy program has been shown to be mediated by various signaling pathways, such as the calcineurin/NFAT (nuclear factor of activated T-cells) pathway ${ }^{5}$ and c-Src-mediated pathways. ${ }^{6-9}$ We have recently shown that c-Src activity is inversely correlated with calreticulin expression level. To date, no studies have been undertaken to assess the mechanism of calreticulin action during cardiac hypertrophy. In fact, only two previous studies exist in which the authors examined calreticulin in in vivo models of cardiac hypertrophy, and only calreticulin levels were assessed in these studies. ${ }^{10,11}$ One study documents an increase in calreticulin levels after hypertrophic induction by calsequestrin-overexpression in mice, ${ }^{10}$ whereas the other study shows a modest increase in calreticulin levels after induction of hypertrophy in rats. ${ }^{11}$ Thus, in this study, our goal was not only to investigate the effects of transverse aortic constriction (TAC) on the levels of calreticulin but also to examine the levels of c-Src, a tyrosine kinase involved in important adhesive changes that occur during the hypertrophic program. Furthermore, our goal was to investigate the effects of soluble hypertrophic agonists on embryonic stem (ES) cell-derived cardiomyocytes that were devoid of calreticulin. In light of the fact that calreticulin levels are decreased in end-stage human heart failure, ${ }^{12}$ it is interesting to speculate that increased calreticulin levels may serve to be beneficial in preventing heart failure. This study, then, is a crucial first step toward understanding the mechanism by which an endoplasmic reticulum-resident protein is able to drastically influence cardiac pathology, including the cardiac hypertrophic program.

\section{Materials and Methods}

\section{Transverse Aortic Constriction of Mice}

Ten- to 12-week-old male CD1 mice (25 to $30 \mathrm{~g}$ ) and C57BL/6 mice (20 to $25 \mathrm{~g}$ ) were used in this study. A minimum of six mice were subjected to TAC at the aortic arch between the brachiocephalic and left common carotid arteries to induce left ventricular hypertrophy. A minimum of six sham mice were used per time period, and these mice were subjected to the same conditions as the TAC mice (anesthetic, surgery-except for the aortic coarctation, food, and housing). Sham mice underwent a comparable operation in which the aortic arch was isolated and a band was placed around the aorta but was not ligated and was removed. The time points for the banding and sham experiments were 7 days, 15 days, and 21 days. One to $1.5 \%$ of isofluorene gas was used as an anesthetic, administered via intratracheal intubation for the surgeries and via a mask for hemodynamic measurements.

\section{Hemodynamic Measurements}

After set time periods of TAC, the mice were subjected to blood pressure measurements by using a Millar Microtip (Millar Instruments, Inc., Houston, Texas) pressure cath- eter $1.4 \mathrm{~F}$. The catheter was inserted through the right carotid artery, pushed into the ascending aorta, and finally into the left ventricle.

\section{Histological Staining}

After periods of TAC, left ventricles were weighed (blotted dry and wet weight taken) and then were either lysed and subjected to SDS-polyacrylamide gel electrophoresis (PAGE) and Western blotting or fixed in $4 \%$ paraformaldehyde for 1 hour for histological analysis. After fixation, the tissues were placed in a $30 \%$ sucrose solution overnight on a shaker at $4^{\circ} \mathrm{C}$. The tissues were then placed in a 1:1 solution of HistoPrep Frozen Tissue Embedding Media (Fisher Scientific, Ottawa, Ontario, Canada): PBS for 8 hours after which they were frozen in $100 \%$ embedding medium by using 2-methylbutane cooled in liquid nitrogen. They were sectioned into $10-\mu \mathrm{m}$ sections by using a Reichart cryostat (Reichert Cryostat, Block Scientific, Inc., Bohemia, NY) at $-27^{\circ} \mathrm{C}$.

\section{Cardiomyocyte Differentiation from ES Cells}

Calreticulin-null R1 ES cell lines ${ }^{13}$ and their wild-type counterparts were used to obtain cardiomyocytes via the hanging drop method. ${ }^{14}$ The wild-type CGR8 cell line ${ }^{15}$ was used as another control. Briefly, 500 ES cells (at passage 2) per $25 \mu \mathrm{l}$ drop of differentiation medium were placed on the lids of tissue culture dishes for 2 days. The differentiation medium consisted of high glucose Dulbecco's modified Eagle's medium (Multicell, Wisent, StBruno, Quebec, Canada), supplemented with 20\% fetal bovine serum (Multicell, Wisent), $2 \mathrm{mmol} / \mathrm{L}$ of L-glutamine (Gibco, Burlington, Ontario, Canada), $1 \mathrm{mmol} / \mathrm{L}$ of sodium pyruvate (Gibco), and $0.1 \mathrm{mmol} / \mathrm{L}$ of minimal essential medium nonessential amino acids (Gibco). After 2 days in hanging drops, the aggregated ES cells, now termed embryoid bodies, were floated in differentiation medium for 3 days. The embryoid bodies were then plated onto tissue culture dishes (coated with $0.1 \%$ gelatin in PBS for 1 hour at $37^{\circ} \mathrm{C}$ ). Spontaneously beating areas could be detected in the calreticulin-null embryoid bodies as early as day 6 (day $0=$ making of hanging drops).

\section{Isolation of Cardiomyocytes on a Percoll Gradient}

Embryoid bodies at day 10 to day 20 of development were digested with $3 \mathrm{mg} / \mathrm{ml}$ of collagenase II (Gibco, Invitrogen, Burlington and Oakville, Ontario, Canada) and $1 \mathrm{mg} / \mathrm{ml}$ of pancreatin (Sigma) for $3 \times 30$ minutes at $37^{\circ} \mathrm{C}$. Fetal bovine serum was then added to end the enzymatic reaction, and the cells were centrifuged at room temperature at $3000 \mathrm{rpm}$ for 6 minutes. The pellet was then dissolved in 1 X ADS buffer (containing $116 \mathrm{mM}$ $\mathrm{NaCl}, 20 \mathrm{mM}$ HEPES, $1 \mathrm{mM} \mathrm{NaH} \mathrm{PO}_{4}, 5.5 \mathrm{mM}$ glucose, $5 \mathrm{mM} \mathrm{KCl}$ and $0.4 \mathrm{mM} \mathrm{MgSO}_{4}$ ) and loaded onto a Percoll gradient (Amersham Biosciences, Baie d'Urfe, Quebec, Canada) consisting of two density layers: 0.9 and 0.7 . The cells were then centrifuged at room temperature at $30,000 \times g$ for 30 minutes. The cardiomyocyte layer was 
Table 1. PCR Reaction Conditions

\begin{tabular}{|c|c|c|c|c|c|}
\hline Gene & Forward primer & Reverse primer & $\begin{array}{c}\text { Annealing } \\
\text { temperature, }{ }^{\circ} \mathrm{C}\end{array}$ & $\begin{array}{c}\text { Cycle } \\
\text { number }\end{array}$ & $\begin{array}{l}\text { Product } \\
\text { size, bp }\end{array}$ \\
\hline L32 & 5'-CATGGCTGCCCTTCGGCCTC-3' & 5'-CATTCTCTTCGCTGCGTAGCC-3' & 55 & 22 & 403 \\
\hline$\beta-M H C$ & 5'-GCCAACACCAACCTGTCCAAGTTC- $3^{\prime}$ & $5^{\prime}-\mathrm{TTCAAAGGCTCCAGGTCTCAGGGC-3^{ \prime }}$ & 55 & 26 & 202 \\
\hline Nkx 2.5 & 5'-CATTTTACCCGGGAGCCTACGGTG-3' & 5'-GCTTTCCGTCGCCGCCGTG-3' & 55 & 26 & 300 \\
\hline GATA-4 & $5^{\prime}-$ CCTCTCCCAGGAACATCAAA - $3^{\prime}$ & 5'-ACCCATAGTCACCAAGGCTG-3' & 55 & 29 & 197 \\
\hline
\end{tabular}

found to be in the boundary between the two Percoll density layers. The cardiomyocytes were collected from the Percoll gradient and plated onto gelatinized dishes $(0.5 \%$ gelatin in PBS) in differentiation medium as described in the previous paragraph.

\section{Endothelin-1 and Phenylephrine Treatment of Cardiomyocytes}

Cardiomyocytes freshly isolated from embryoid bodies were plated for 24 hours on gelatinized plates. They were then serum starved for 24 hours and treated with 1 $\mu \mathrm{mol} / \mathrm{L}$ of endothelin-1 (ET1; Sigma) for either 48 hours or 4 days or with $100 \mu \mathrm{mol} / \mathrm{L}$ of phenylephrine (PE; Sigma) for 48 hours or 4 days. The c-Src inhibitor, PP2 (4-amino-5-(4-chlorophenyl)-7-(t-butyl)pyrazolo[3,4-day] pyramidine) from Sigma, was also added along with ET1 or PE to some cardiomyocyte cultures at $5 \mu \mathrm{mol} / \mathrm{L}$ for 48 hours. The cardiomyocytes were then subjected to immunofluorescence or SDS-PAGE and Western blotting. Cardiomyocyte cell areas were measured by using Image J (http://rsb.info.nih.gov).

\section{Immunolabeling and Microscopy}

For immunolocalization, cells on coverslips were fixed in $3.7 \%$ formaldehyde in PBS for 10 minutes. After washing (three times for 5 minutes) in PBS, the cells were permeabilized with $0.1 \%$ Triton $X-100$ in buffer containing 100 $\mathrm{mmol} / \mathrm{L}$ of $\mathrm{A}$. piperazine-N, $\mathrm{N}$ '-bis 2-ethanesulfonic acid (PIPES), $1 \mathrm{mMol} / \mathrm{L}$ of EGTA, and $4 \%$ (w/v) of polyethylene glycol 8000 ( $\mathrm{pH} \mathrm{6.9)}$ for 2 minutes, and the cells were washed again (three times for 5 minutes in PBS). Texas Red phalloidin (Invitrogen, Molecular Probes, Burlington, ON, Canada) was used at a concentration of 1:10 to visualize the actin cytoskeleton. After the final wash (three times for 5 minutes), the slides were mounted in Vinol 205S, which contained $0.25 \%$ of 1,4 -diazabicyclo-(2,2,2)-octane and $0.002 \%$ of $\mathrm{p}$-phenylenediamine to prevent photobleaching. A Bio-Rad MRC-600 confocal fluorescence microscope equipped with a krypton/argon laser was used for fluorescence and phase contrast microscopy.

\section{SDS-PAGE and Western Blotting}

Mouse hearts were homogenized in lysis buffer containing $0.5 \%$ of NP-40, as well as $10 \mu \mathrm{l} / \mathrm{ml}$ of phosphatase inhibitor cocktail (Sigma) and $10 \mu \mathrm{l} / \mathrm{ml}$ of protease inhibitor cocktail (Sigma). For in vitro studies, embryoid bodies were treated with trypsin/EDTA (Sigma) and pipetted in collagenase type II (Gibco, Invitrogen) until a single cell solution was achieved. The cells were then counted by using a series Z1 Beckman Coulter Counter (Mississauga, Ontario, Canada) and lysed. Protein samples were quantified by using the Bio-Rad Protein Assay (Bio-Rad Laboratories) according to the manufacturer's instructions. Finally, protein samples were subjected to SDS-PAGE and Western blotting as described by Mery et al. ${ }^{16}$ The primary antibodies were used at the following dilutions in Tris-buffered saline supplemented with $0.1 \%$ Tween 20: goat anti-calreticulin (gift from Dr. Michalak), 1:300; rabbit anti-glyceraldehyde3-phosphate dehydrogenase (GAPDH; Lab Frontier, Burlington, Ontario, Canada), 1:2000; mouse anti-cardiac MHC (Cedarlane Laboratories Limited, Burlington, Ontario, Canada), 1:1000; mouse anti-MLC2v ventricular myosin light chain 2; (Synaptic Systems, Goettingen, Germany), 1:1000; mouse anti-PAN cadherin (Sigma), 1:1000; mouse anti-active Src (BioSource, Burlington, Ontario, Canada), 1:1000; mouse anti-Src pY418 (BioSource), 1:1000; mouse anti-c-Src (Upstate Cell Signaling Solutions, Pickering, Ontario, Canada), 1:1000; and mouse anti-vinculin (Sigma), 1:1000. Mouse anti-skeletal $\alpha$-actin was made by S. Clement. All secondary antibodies were conjugated to horseradish peroxidase and used at a dilution of 1:10000 (Jackson ImmunoResearch Laboratories, West Grove, PA). The protein bands were visualized by using the ECL-Plus Detection System (Amersham).

\section{Semiquantitative RT-PCR}

mRNA expression levels were determined by semiquantitative RT-PCR as described previously. ${ }^{17}$ Total RNA was isolated with TRIzol Reagent (Sigma) according to the manufacturer's instructions. Chloroform was then added, and the solution was centrifuged at $12,000 \times g$ for 15 minutes at $4^{\circ} \mathrm{C}$ and the aqueous phase was transferred to a fresh tube. The RNA was precipitated with isopropyl alcohol overnight at $4^{\circ} \mathrm{C}$, pelleted by centrifugation at $12,000 \times g$ for 10 minutes at $4^{\circ} \mathrm{C}$, washed once with $75 \%$ ethanol, vortexed, and centrifuged at $7500 \times g$ for 5 minutes at $4{ }^{\circ} \mathrm{C}$, and the RNA pellet was briefly air-dried $(2$ minutes). RNA was dissolved in $50 \mu$ l of autoclaved distilled water by incubating for 10 minutes at $55^{\circ} \mathrm{C}$ and stored at $-80^{\circ} \mathrm{C}$ until use. The RNA concentration of each sample was measured by a spectrophotometer (DU640, Beckman) at $260 \mathrm{~nm}$. Total cellular RNA was reverse transcribed with a Superscript II first strand cDNA synthesis kit (Invitrogen) according to the manufacturer's directions. PCR was performed with a PCR kit (Qiagen, Mississauga, Ontario, Canada) according to the manufacturer's directions. The primer sequences and conditions used for each PCR reaction are in Table 1. The PCR 
Table 2. Hemodynamic Measurements of CD1 Mice

\begin{tabular}{|c|c|c|c|c|c|c|c|c|c|}
\hline \multirow[b]{2}{*}{ Parameter } & \multicolumn{3}{|c|}{ Day 7} & \multicolumn{3}{|c|}{ Day 15} & \multicolumn{3}{|c|}{ Day 21} \\
\hline & Sham & Banded & $P^{*}$ & Sham & Banded & $P^{*}$ & Sham & Banded & $P^{*}$ \\
\hline$N$ & 3 & 5 & & 2 & 4 & & 3 & 5 & \\
\hline Heart rate, beats per min & $418 \pm 43$ & $435 \pm 56$ & NS & $493 \pm 53$ & $520 \pm 80$ & NS & $469 \pm 49$ & $490 \pm 55$ & NS \\
\hline $\begin{array}{l}\text { Left ventricular weight/body weight } \\
\text { ratio, } \times 1000\end{array}$ & $3.3 \pm 0.06$ & $4.2 \pm 0.4$ & 0.0029 & $3.3 \pm 0.1$ & $4.8 \pm 0.4$ & 0.0025 & $3 \pm 0.04$ & $5 \pm 0.9$ & 0.008 \\
\hline $\mathrm{AP}_{\text {sys }}, \mathrm{mmHg}$ & $105 \pm 0.4$ & $169 \pm 9$ & 0.00001 & $95 \pm 5$ & $135 \pm 21$ & 0.026 & $98 \pm 16$ & $176 \pm 2.8$ & 0.0003 \\
\hline LVP $_{\text {sys }}, \mathrm{mmHg}$ & $101 \pm 3$ & $139 \pm 26$ & 0.015 & $98 \pm 6$ & $129 \pm 17$ & 0.035 & $102 \pm 7$ & $181 \pm 10$ & 0.00003 \\
\hline$+\mathrm{dP} \mathrm{dt}_{\max }, \mathrm{mmHg} / \mathrm{s}$ & $14,442 \pm 2989$ & $11,853 \pm 2986$ & NS & $11,307 \pm 288$ & $10,129 \pm 667$ & 0.04 & $9391 \pm 1305$ & $9474 \pm 1402$ & NS \\
\hline$-\mathrm{dP}_{\mathrm{dt}} \mathrm{dt}_{\mathrm{in}}, \mathrm{mmHg} / \mathrm{s}$ & $-12,164 \pm 3075$ & $-9660 \pm 33,338$ & NS & $-10,117 \pm 667$ & $-9065 \pm 989$ & NS & $-7865 \pm 1484$ & $-9320 \pm 1932$ & NS \\
\hline$\left(+\mathrm{dP} / \mathrm{dt}_{\max }\right) /\left(-\mathrm{dP} / \mathrm{dt}_{\min }\right)$ & $1.19 \pm 0.97$ & $1.23 \pm 0.89$ & & $1.12 \pm 0.43$ & $1.12 \pm 0.67$ & & $1.19 \pm 0.88$ & $1.02 \pm 0.73$ & \\
\hline
\end{tabular}

$A P_{\text {sys }}=$ systolic aortic pressure, $\mathrm{LVP}_{\text {sys }}=$ systolic left ventricular pressure, $\mathrm{dP}=$ derivative of pressure, $\mathrm{dt}_{\min }=$ minimum time derivative, $\mathrm{dt}_{\text {max }}=$ maximum time derivative. ${ }^{*}$ Data presented as mean \pm SD. NS, not significant.

products were run in $1.5 \%$ agarose gel for 28 minutes at $100 \mathrm{~V}$, stained with ethidium bromide, and visualized under Bio-Rad Gel Doc 2000. Equal amounts of cDNA were used in each reaction sample by first normalizing band intensity to that for the housekeeping gene, ribosomal protein L32. The intensities of amplified bands were quantified by using ImageQuant (GE Healthcare, formerly Amersham Biosciences, Baie d'Urfe, Quebec, Canada) software (version 1.2) and normalized to L32.

\section{Statistical Analysis of the Data}

SDs for replicate data were calculated and displayed as error bars. Differences between mean values for different treatments were calculated by using the two-tailed un- paired Student's $t$ test and were considered to be significant at $P<0.05$.

\section{Results}

Induction of Left Ventricular Hypertrophy in Both CD1 and C57BL/6 Mice after TAC

To investigate the hypertrophic response in CD1 and C57BL/6 mice after TAC, physiological measurements and histological staining were performed. For all experimental periods examined in CD1 mice, namely 7 days, 15 days, and 21 days, the left ventricular weight to body weight ratios were significantly increased in the TAC
A

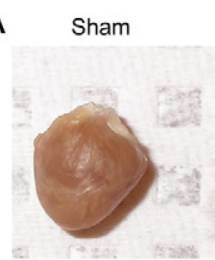

C

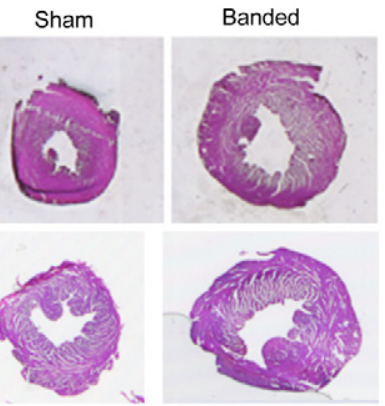

G

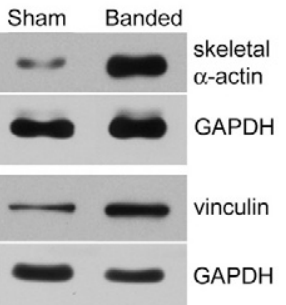

Banded

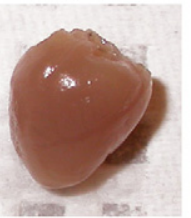

E

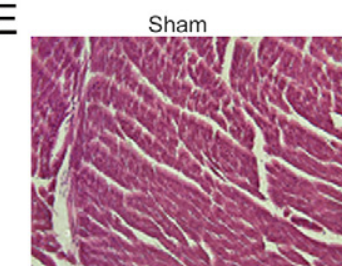

F
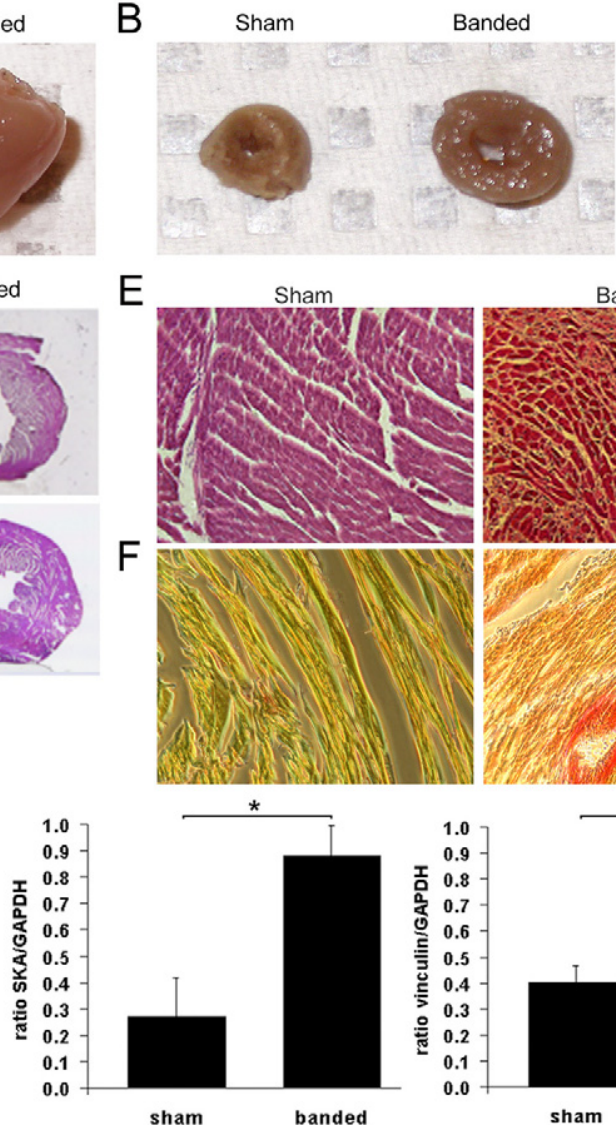

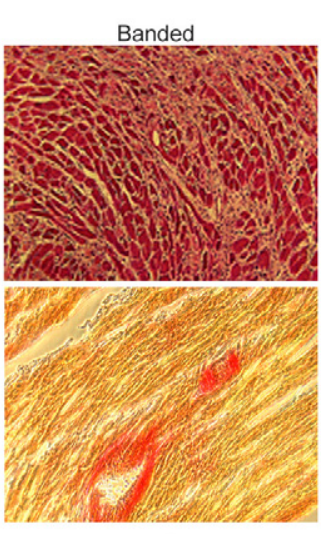

*

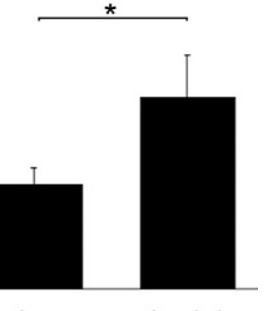

Figure 1. An aortic banding increases heart and ventricle size and reinitiates the fetal cardiac program. A: Gross morphology of $\mathrm{C} 57 \mathrm{BL} / 6$ mouse whole left ventricles of control (sham) versus TAC hearts. B: Representative mid crosssections of the left ventricle from sham and TAC hearts. C and D: H\&E stained cross-sections of left ventricles of $\mathrm{CD} 1 \mathrm{mice}(\mathbf{C})$ and $\mathrm{C} 57 \mathrm{BL} / 6$ mice (D) after 21 days of sham versus TAC. Note the increased size and wall thickness of the banded hearts. E: H\&E stained longitudinal section of the left ventricles after 7 days of aortic banding Note that as early as day 7 , there is interstitial fibrosis in the banded hearts. F: Representative cross-section of $\mathrm{C} 57 \mathrm{BL} / 6$ left ventricles after picrosirius red staining. After banding, there is a slight increase in collagen deposition, as indicated by the red staining. G: By Western blotting, the levels of both skeletal $\alpha$-actin and vinculin were higher after TAC (21 days) of C57BL/6 mice. Protein levels were normalized to GAPDH. Error bars indicate SDs $\left({ }^{*} P<0.05 ; n=\right.$ 12 per condition). SKA, skeletal $\alpha$-actin. 
(banded) versus sham mice (Supplemental Figure S1A, see http://ajp.amjpathol.org). C57BL/6 mice were banded for 21 days and also exhibited a marked increase in left ventricular weight/body weight ratio (Supplemental Figure S1B, see http://ajp.amjpathol.org). Hemodynamic measurements revealed that both aortic systolic and left ventricular systolic pressures were significantly higher in banded versus sham mice at all time periods examined in both strains of mice (Supplemental Figure S1, C-F, see http://ajp.amjpathol.org). Diastolic pressures were not affected by TAC (data not shown). Other physiological parameters of the CD1 mice are listed in Table 2.

Gross morphological (Figure 1, A and B) and histological sections (Figure 1, C-F) of left ventricles of both mouse strains revealed a significant increase in the total size and wall thickness of the left ventricles of banded mice compared with sham mice. H\&E staining of left ventricular sections further revealed that as early as day 7 , there was marked interstitial fibrosis present in the banded, but not the sham, ventricles of CD1 mice (Figure 1E). Interstitial fibrosis is a key change that occurs in pressure overload hypertrophy. ${ }^{18,19} \mathrm{C} 57 \mathrm{BL} / 6$ mice also exhibited a slight increase in collagen deposition into the interstitium after banding, as visualized by picrosirius red staining (Figure 1F).

Lastly, we examined the expression of skeletal $\alpha$-actin in banded versus sham C57BL/6 mice (12 mice were used for each biological replicate) because there is usually re-expression of the fetal gene program after TAC. For example, three muscle $\alpha$-actin isoforms are sequentially expressed during cardiac development in vivo, namely smooth muscle $\alpha$-actin, skeletal $\alpha$-actin, and cardiac $\alpha$-actin. ${ }^{20}$ Indeed, we show here that the expression of skeletal $\alpha$-actin was significantly increased after TAC (Figure 1G), suggesting a re-activation, at least in part, of the fetal gene program. Interestingly, the levels of vinculin, a component of both costameres and intercalated disks of cardiomyocytes, were also noticeably elevated after TAC (Figure 1G).

\section{Calreticulin Expression Increases after TAC, Which May Impinge on c-Src-Mediated Signaling Pathways}

Postnatal heart-targeted overexpression of calreticulin causes marked myocardial remodeling leading to marked ventricular dilation. ${ }^{1,2}$ What is the abundance of calreticulin in the heart that undergoes TAC-induced remodeling? To establish the effect of TAC on calreticulin expression, mouse left ventricles were analyzed by Western blotting at day 7,15 , and 21 after the banding. As early as 7 days after surgery, calreticulin levels were elevated in banded versus sham CD1 animals (Figure 2, A and B). Calreticulin levels remained high in TAC ventricles at 15 and 21 days after surgery (Figure 2, A and B). We observed a similar increase in calreticulin levels in C57BL/6 animals after 21 days of TAC (Figure 2, $C$ and D); hence, the effect was maintained across different mouse strains. These data strongly suggest that calreticulin is induced after TAC, with vinculin following the same trend.
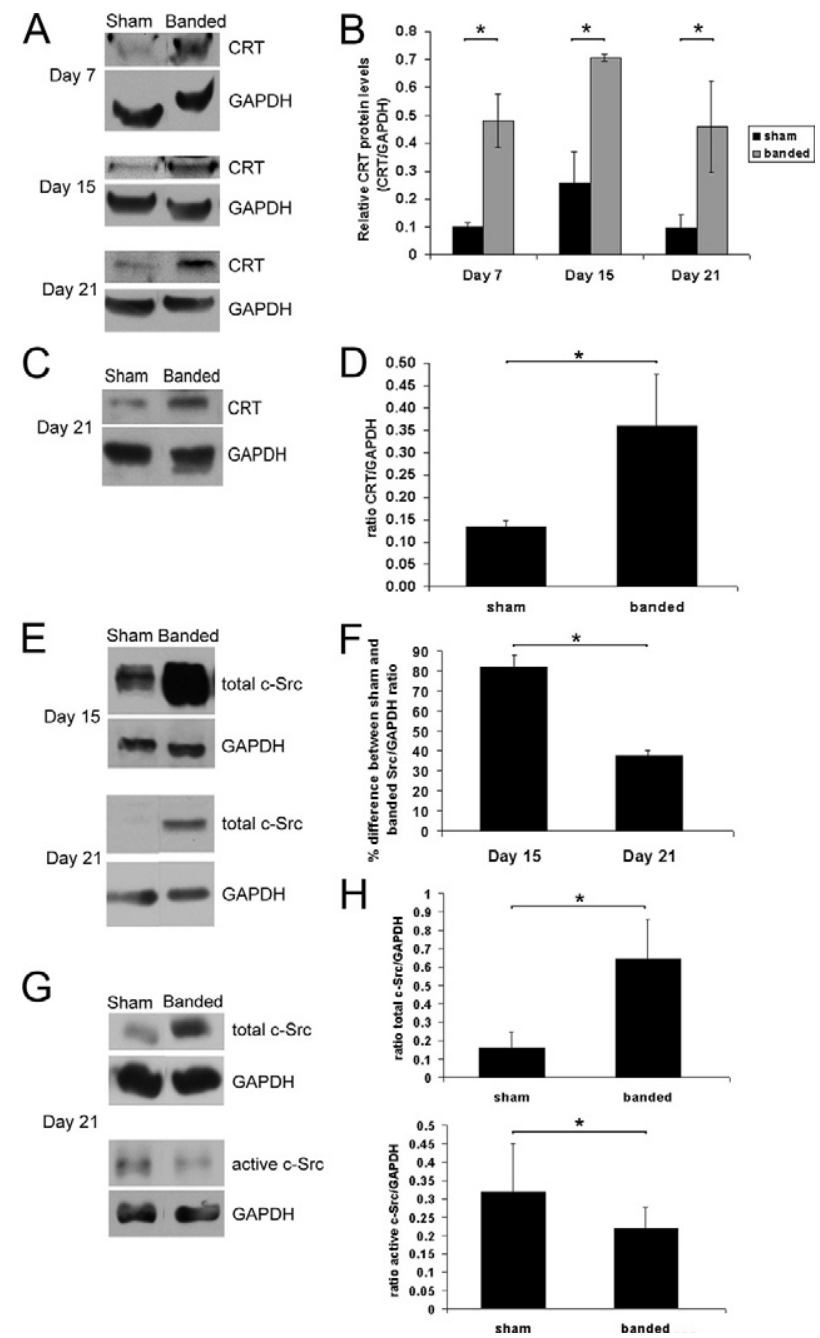

Figure 2. Calreticulin and c-Src levels are elevated after aortic banding Western blots of calreticulin (CRT) levels at day 7, 15, and 21 after TAC versus sham in CD1 mice (A). B: Quantification of calreticulin protein levels obtained by Western blotting for each banding time period shown in $\mathbf{A}$, as a ratio to GAPDH for CD1 mice $\left({ }^{*} P<0.05 ; n=7\right)$. C: Western blots of CRT levels at day 21 after TAC versus sham in C57BL/6 mice are shown. D: Quantification of calreticulin protein levels obtained by Western blotting as shown in $\mathbf{C}$, as a ratio to GAPDH for $\mathrm{C} 57 \mathrm{BL} / 6$ mice $\left({ }^{*} P<0.05 ; n=6\right)$. E: Total c-Src levels in TAC versus sham CD1 mice at 15 and 21 days after the banding are shown. F: Relative ratio (shown as the percent difference) of total c-Src levels between sham and banded mice for each banding time period shown in E. G: Total c-Src and active c-Src (phosphorylated on Y418) in C57BL/ 6 mice after 21 days of TAC are shown. H: Relative levels of total C-Src (top) and active C-Src (bottom) at day 21 after TAC corresponding to $\mathbf{G}\left({ }^{*} P<0.05 ; n=6\right)$. Error bars in $\mathbf{B}, \mathbf{D}, \mathbf{F}$, and $\mathbf{H}$ indicate SDs.

A protein shown by several groups to be involved in the hypertrophic response is C-Src. ${ }^{6-9}$ As with vinculin, we have previously shown c-Src activity to be correlated with calreticulin levels, thereby influencing cell-substratum adhesion. ${ }^{21,22}$ However, unlike vinculin, c-Src activity levels are inversely correlated with calreticulin levels. To investigate the effects of aortic banding on c-Src levels, Western blotting was performed on mouse left ventricles for total c-Src levels (Figure 2E). At 15 days after TAC, the banded CD1 animals contained substantially higher amounts of c-Src compared with the sham mice. Importantly, c-Src levels were distinctly lower in the banded animals after 21 days of TAC compared with 15 days, and 
A

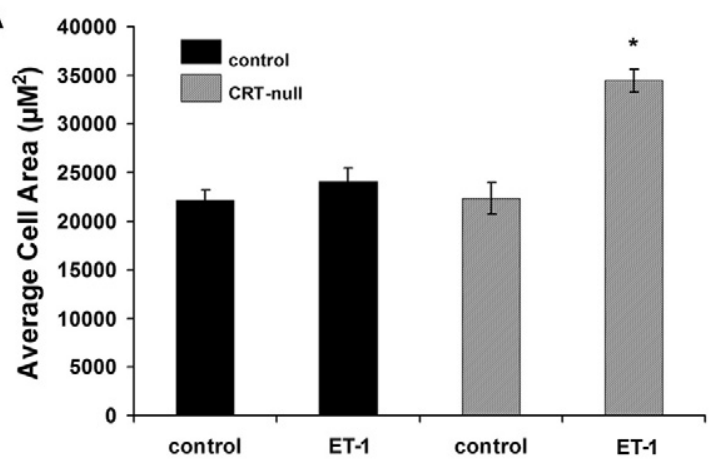

B

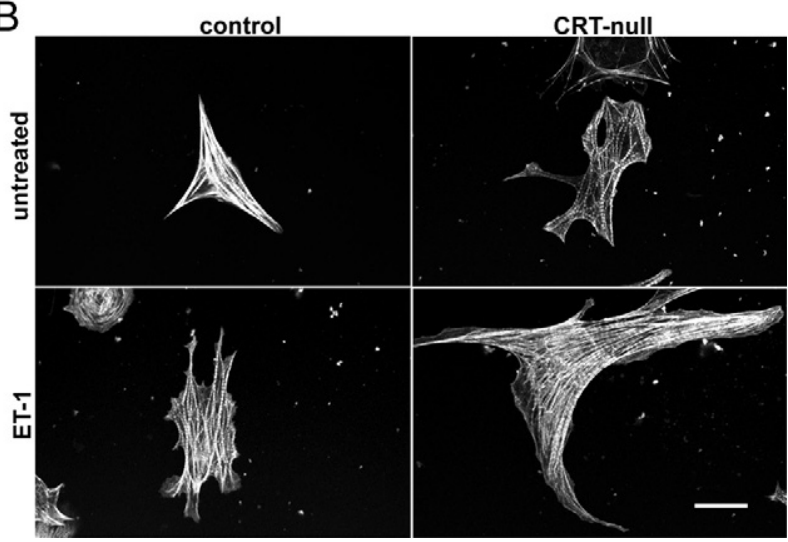

Figure 3. ET1 treatment induces hypertrophy in calreticulin-null cardiomyocytes. A: Cell area measurements of cardiomyocytes isolated by Percoll gradient centrifugation from wild-type (control) and calreticulinnull (CRT-null) embryoid bodies at day 14 of the cardiac differentiation and subsequently treated with $1 \mu \mathrm{mol} / \mathrm{L}$ of ET1 for 4 days. Error bars in indicate SDs ( $\left.{ }^{*} P<0.05 ; n=6\right)$. B: Fluorescence images of cardiomyocytes labeled with Texas Red phalloidin to demonstrate the actin-containing contractile apparatus. Scale bar $=25 \mu \mathrm{m}$.

the actual difference in c-Src levels between sham and banded animals dramatically decreased by this period of time (Figure 2F). Figure 2G shows that, after 21 days of TAC, compared with total c-Src abundance, active c-Src levels (c-Src phosphorylated on tyrosine 418) were much lower in the banded animals compared with sham (Figure 2 , $G$ and $H)$. In summary, there seems to be an inverse correlation between calreticulin and active c-Src levels after TAC, as well as with increased duration of banding.

\section{The Effects of ET1 and PE on Cardiomyocytes Derived from Embryoid Bodies Differentially Expressing Calreticulin}

To further investigate the role of calreticulin in cardiac hypertrophy, we wanted to use a calreticulin knockout strategy. However, because calreticulin-null embryos are embryonic lethal at embryonic day 14.5 , banding studies could not be performed on these mice. Thus, cardiomyocytes derived from calreticulin-null embryoid bodies were used. To examine the degree of hypertrophy, if any, exhibited by calreticulin-null versus wild-type embryoid bodies, we examined the effects of soluble hypertrophic agonists, ET1 and PE. Treatment with ET1 for 4 days caused a discernible but not sta-
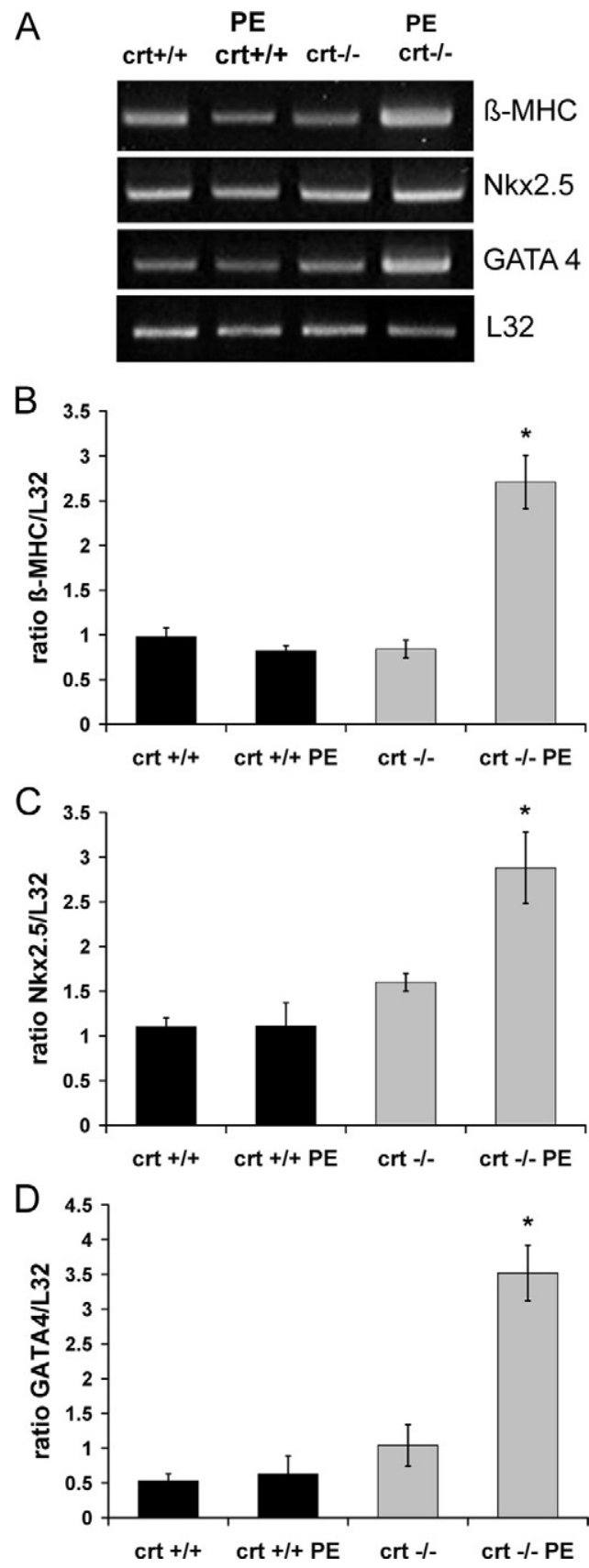

Figure 4. Induction of hypertrophy increases cardiac MHC and transcription factors expression. A: mRNA expression of cardiac $\beta$-MHC and cardiac-specific transcription factors, Nkx 2.5 and GATA-4, from wild-type $\left(\mathrm{crt}^{+/+}\right)$and calreticulin-null $\left(\mathrm{crt}^{-1-}\right)$ cardiomyocytes with or without PE treatment. The cells were purified from embryoid bodies at day 14 of the cardiac differentiation by Percoll gradient centrifugation. B: Relative expression of $\beta$-MHC $\left({ }^{*} P<0.01 ; n=5\right)$ is shown. C: Relative expression of Nkx2.5 (* $P<0.01 ; n=5)$ is shown. D: Relative expression of GATA- $4\left({ }^{*} P<0.01 ; n=5\right)$ is shown. All samples were normalized to the housekeeping gene L32. Error bars in $\mathbf{B}-\mathbf{D}$ indicate SDs.

tistically significant increase in the cell area of the wild-type cardiomyocytes, and treatment with ET1 caused a substantial and statistically significant increase in the cell area of the calreticulin-null cardiomyocytes (Figure 3, A and B). We also examined the effects of another soluble hypertrophic agonist, PE. Treatment with PE increased mRNA levels of cardiac $\mathrm{MHC}$ and two cardiac-specific transcription factors, 

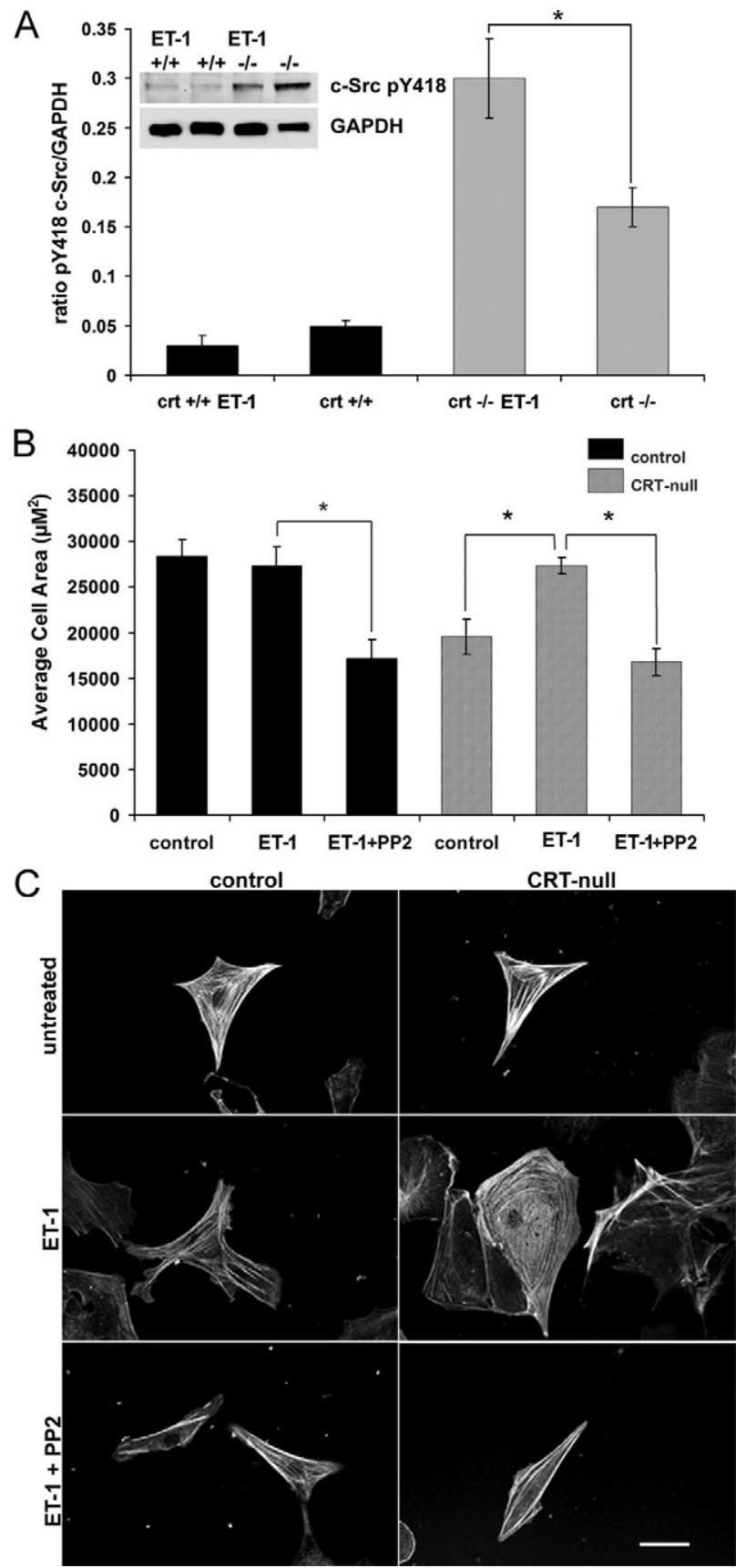

Figure 5. c-Src inhibition antagonizes cardiomyocyte hypertrophy induced by ET1. A: Western blot of c-Src phosphorylated on tyrosine 418 (c-Src pY 418) in cardiomyocytes derived by Percoll gradient centrifugation from embryoid bodies at day 11 of cardiac development is shown. c-Src activity is higher in calreticulin-null cardiomyocytes $(-/-)$ versus wild-type cells $(+/+)$ and it increases after ET1 treatment. GAPDH was used a loading control. Graph shows quantification of c-Src levels. Error bars represent SD (* $P<0.05 ; n=6)$. B: Cell area measurements of cardiomyocytes isolated from day 14 embryoid bodies derived by Percoll gradient centrifugation and treated for 48 hours with ET1 alone or with ET1 plus the c-Src inhibitor, PP2. After 48 hours of ET1 treatment (1 $\mu \mathrm{mol} / \mathrm{L}$ ), calreticulin-null (CRT-null) cardiomyocytes exhibited a greater increase in cell size compared with control cells. Treatment with the c-Src inhibitor, PP2 $(5 \mu \mathrm{mol} / \mathrm{L})$, abolished the increase in cell size of calreticulin-null cardiomyocytes seen with ET1 treatment. Error bars in A and B indicate SDs $\left({ }^{*} P<0.05 ; n=6\right)$. C: Fluorescence images of cells labeled with Texas Red phalloidin to reveal cell size and the actin thin filaments within the contractile apparatus after ET1 and ET1/PP2 treatment in control and CRT-null cardiomyocytes are shown. Scale bar $=25 \mu \mathrm{m}$.
Nkx2.5 and GATA4, in the calreticulin-null cardiomyocytes (Figure 4, A-D). mRNA levels of these proteins factors were unchanged in the wild-type cardiomyocytes after PE treatment. Thus, we conclude that the calreticulin-null cardiomyocytes are able to elicit a greater hypertrophic response to soluble mediators compared with wild-type cells.

\section{c-Src Inhibition Diminishes the Hypertrophic Response Elicited by ET1 in Calreticulin-Null Cardiomyocytes}

Since calreticulin-null cardiomyocytes elicited a significantly greater hypertrophic response to ET1 than the wild-type cells, we explored the mechanism by which calreticulin was able to down-regulate the response to hypertrophic signals. Given that c-Src seems to be a modulator of the hypertrophic response and that c-Src levels are gradually attenuated during TAC, we examined the effects of c-Src inhibition on the hypertrophic response induced by ET1. Firstly, we found that calreticulin-null cardiomyocytes derived from ES cells had increased levels of c-Src phosphorylated on tyrosine 418 (src pY418), indicative of active c-Src (Figure 5A). c-Src contains an autophosphorylation site at tyrosine 418 , which renders the kinase active when phosphorylated. ${ }^{23}$ Active c-Src levels further increased in calreticulin-null cardiomyocytes after ET1 treatment. Figure 5, B and C, shows that the increase in cell area seen in calreticulin-

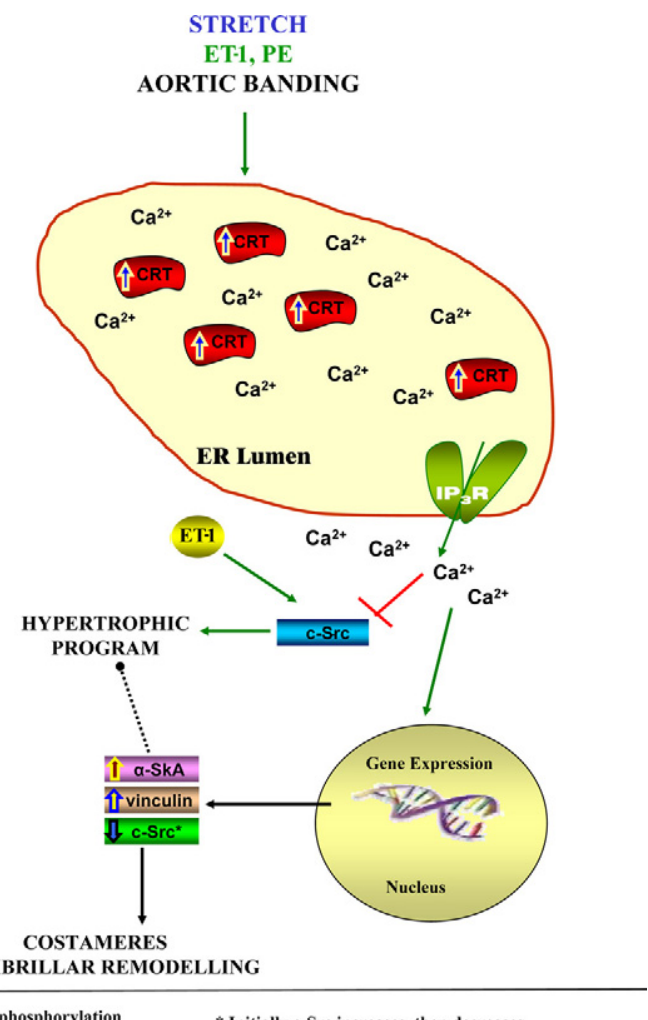

(C) dephosphorylation

SKA - a-skeletal actin

Initially c-Sre increases, then decrease

Figure 6. Schematic representation of the effects of calreticulin (CRT) on cardiomyocyte responses to hypertrophic signals and stretch and aortic banding. 
null cells after ET1 treatment is diminished on treatment with the c-Src inhibitor, PP2. As Shu and Shayman ${ }^{24}$ have shown, PP2 causes dephosphorylation of c-Src on tyrosine 418 , while it also causes phosphorylation of c-Src on tyrosine 529. Together, these events inhibit the tyrosine kinase activity of c-Src.

\section{Discussion}

This study shows that calreticulin is up-regulated after the induction of pressure overload hypertrophy by coarctation of the aortic arch of CD1 and C57BL/6 mice. The signaling pathways underlying cardiac hypertrophy parallel those of the cardiac developmental program. Many fetal genes, such as $\beta-\mathrm{MHC}$, atrial natriuretic protein, and skeletal $\alpha$-actin are re-expressed during the cardiac hypertrophic response. ${ }^{25}$ Indeed, we observed the up-regulation of skeletal $\alpha$-actin in mice after aortic coarctation. Since calreticulin has been shown by us to be up-regulated during heart development, ${ }^{26}$ it is not surprising that it would also be up-regulated during the hypertrophic response, as we have found here. However, it is important to discern whether calreticulin is up-regulated as a part of the hypertrophic response, or if its up-regulation is protective against cardiac hypertrophy.

Our working hypothesis has initially been that calreticulin, in line with other genes in cardiac development, is up-regulated to augment the hypertrophic response. Our work on ES-cell derived cardiomyocytes, however, suggests the opposite (ie, that calreticulin attenuates the hypertrophic response). Given that calreticulin is a stress protein, ${ }^{27}$ this study was focused on verifying our new hypothesis that it is the "protective" function of calreticulin that is invoked during the induction of hypertrophy. In summary, as virtually nothing is known about the role of calreticulin in the hypertrophic heart, the specific and novel objective of this work was to begin to unravel the role of calreticulin in cardiac hypertrophy. To this end, in vivo analyses of calreticulin-null versus wild-type mice would have provided important clues. However, since calreticulin-null mice are embryonic lethal due to severe cardiac defects (26), such studies are not feasible. For this study, we used cardiomyocytes derived from ES cells to analyze the hypertrophic response of calreticulin-null and wild-type cells to soluble hypertrophic agonists. One such soluble hypertrophic agonist is ET1, a vasoconstrictor, which has a potent stimulatory effect on cardiomyocytes. ${ }^{28} \mathrm{ET} 1$ has been shown to stimulate tyrosine kinase activity, and specifically, it has been shown to activate c-Src. ${ }^{7,29,30}$ Activated c-Src was further shown to participate in the induction of atrial natriuretic protein gene expression in cardiomyocytes after ET1 treatment. ${ }^{7}$ In addition, ET1 induction of the immediate early gene, $c$-fos, required activated c-Src. ${ }^{29,30}$ This may explain the greater response to ET1 of calreticulin-null cardiomyocytes, which have higher c-Src activity than their wild-type counterparts. It should also be kept in mind that, as opposed to other species, mouse cardiomyocytes, when put in vitro undergo spontaneous hypertrophy and only weakly (if at all) respond to hypertrophic stimuli, PE included. . $^{31,32}$
Our main hypothesis emerging from this study is that calreticulin may attenuate the hypertrophic response by down-regulating total c-Src levels, as well as by effectively decreasing c-Src Y418 phosphorylation/activation. Although c-Src levels are initially higher in banded mice compared with sham mice, longer periods of banding show a dampening of this initial increase in total C-Src levels. Thus, it seems that there may be a threshold level of calreticulin or a threshold period of time that calreticulin is increased, before the attenuation of total c-Src levels can occur. In addition, we see decreased phosphorylated/ active $\mathrm{c}-\mathrm{Src}$ by 21 days of banding, suggesting that by this time, calreticulin is able to start attenuating, at least in part, the hypertrophic response. It must be kept in mind that our current data are limited to 21 days of TAC, and additional studies will need to be performed to elucidate the effects of long-term TAC (6 to 9 weeks of TAC) on $\mathrm{c}-\mathrm{Src}$ levels and activity. Presently, we can only speculate that, once left ventricular hypertrophy dilation and dysfunction has been established, c-Src activity might subside, while calreticulin expression would remain elevated. Interestingly, Nakamura et al ${ }^{1,2}$ showed that germline constitutive calreticulin overexpression is lethal and that these mice die within 1 to 2 months after birth. To reconcile this apparent controversy with data presented here, we wish to emphasize that Nakamura et al's ${ }^{1,2}$ mice were not induced to overexpress calreticulin as was the case here, but were overexpressers in utero. This is an important point and one that needs to be further investigated because it presents evidence for different roles for calreticulin during development compared with postdevelopment. It seems that calreticulin overexpression is detrimental if its levels are kept high in the heart into the postpartum period, whereas high calreticulin levels seems protective in the face of pressure overload on the heart or in the presence of cardiac hypertrophic agonists. Further in vivo studies with calreticulin over/underexpressing mice will be necessary to validate this hypothesis.

In summary, calreticulin-null cardiomyocytes, with increased c-Src tyrosine kinase activity, may be in a better position to activate fetal genes, albeit without the regulatory control of calreticulin. This impinges on both developmental and hypertrophic signaling pathways, resulting in calreticulin-null cardiomyocytes, which exhibit a greater hypertrophic response than wild-type cells. We show here for the first time that calreticulin, which is accepted to be a key integrator centrally located in the $\mathrm{ER}$, is able to control both gene expression and kinase (c-Src) phosphorylation during the response to various hypertrophic stimuli (Figure 6). It seems, then, that calreticulin has emerged as a pivotal player in the control and attenuation of the cardiac hypertrophic response, which is a novel role for this protein.

\section{Acknowledgments}

We thank Xiaochu Zhang for technical assistance with the histological sectioning and staining. The anti-calreticulin antibodies were a generous gift from Dr. Marek Michalak. 


\section{References}

1. Nakamura K, Robertson M, Liu G, Dickie P, Nakamura K, Guo JQ, Duff HJ, Opas M, Kavanagh K, Michalak M: Complete heart block and sudden death in mice overexpressing calreticulin. J Clin Invest 2001, 107:1245-1253

2. Hattori K, Nakamura K, Hisatomi Y, Matsumoto S, Suzuki M, Harvey RP, Kurihara H, Hattori S, Yamamoto T, Michalak M, Endo F: Arrhythmia induced by spatiotemporal overexpression of calreticulin in the heart. Mol Genet Metab 2007, 91:285-293

3. Hunter JJ, Chien KR: Signaling pathways for cardiac hypertrophy and failure. N Engl J Med 1999, 341:1276-1283

4. Ghatpande S, Goswami S, Mascareno E, Siddiqui MA: Signal transduction and transcriptional adaptation in embryonic heart development and during myocardial hypertrophy. Mol Cell Biochem 1999, 196:93-97

5. Taigen T, De Windt LJ, Lim HW, Molkentin JD: Targeted inhibition of calcineurin prevents agonist-induced cardiomyocyte hypertrophy. Proc Natl Acad Sci USA 2000, 97:1196-1201

6. Kuppuswamy D, Kerr C, Narishige T, Kasi VS, Menick DR, Cooper G: Association of tyrosine-phosphorylated c-Src with the cytoskeleton of hypertrophying myocardium. J Biol Chem 1997, 272:4500-4508

7. Kovacic B, Ilic D, Damsky CH, Gardner DG: c-Src activation plays a role in endothelin-dependent hypertrophy of the cardiac myocyte. J Biol Chem 1998, 273:35185-35193

8. Laser M, Willey CD, Jiang WJ, Cooper G, Menick DR, Zile MR, Kuppuswamy D: Integrin activation and focal complex formation in cardiac hypertrophy. J Biol Chem 2000, 275:35624-35630

9. Torsoni AS, Constancio SS, Nadruz W Jr, Hanks SK, Franchini KG: Focal adhesion kinase is activated and mediates the early hypertrophic response to stretch in cardiac myocytes. Circ Res 2003, 93:140-147

10. Tsutsui H, Ishibashi Y, Imanaka-Yoshida K, Yamamoto S, Yoshida T, Sugimachi M, Urabe $Y$, Takeshita A: Alterations in sarcoplasmic reticulum calcium-storing proteins in pressure-overload cardiac hypertrophy. Am J Physiol Heart Circ Physiol 1997, 272:H168-H175

11. Sato Y, Ferguson DG, Sako H, Dorn GW2, Kadambi VJ, Yatani A, Hoit BD, Walsh RA, Kranias EG: Cardiac-specific overexpression of mouse cardiac calsequestrin is associated with depressed cardiovascular function and hypertrophy in transgenic mice. J Biol Chem 1998, 273:28470-28477

12. Borlak J, Thum T: Hallmarks of ion channel gene expression in end-stage heart failure. FASEB J 2003, 17:1592-1608

13. Li J, Puceat M, Perez-Terzic C, Mery A, Nakamura K, Michalak M, Krause $\mathrm{KH}$, Jaconi ME: Calreticulin reveals a critical Ca2+ checkpoint in cardiac myofibrillogenesis. J Cell Biol 2002, 158:103-113

14. Hescheler J, Fleischmann BK, Lentini S, Maltsev VA, Rohwedel J, Wobus AM, Addicks K: Embryonic stem cells: a model to study structural and functional properties in cardiomyogenesis. Cardiovasc Res 1997, 36:149-162

15. Nichols J, Evans, Evans EP, Smith AG: Establishment of germ-linecompetent embryonic stem (ES) cells using differentiation inhibiting activity. Development 1990, 110:1341-1348

16. Mery L, Mesaeli N, Michalak M, Opas M, Lew DP, Krause K-H:
Overexpression of calreticulin increases intracellular $\mathrm{Ca}^{2+}$-storage and decreases store-operated $\mathrm{Ca}^{2+}$ influx. J Biol Chem 1996, 271: 9332-9339

17. Bonnelye E, Merdad L, Kung V, Aubin JE: The orphan nuclear estrogen receptor-related receptor alpha (ERRalpha) is expressed throughout osteoblast differentiation and regulates bone formation in vitro. J Cell Biol 2001, 153:971-984

18. Cuspidi C, Ciulla M, Zanchetti A: Hypertensive myocardial fibrosis. Nephrol Dial Transplant 2006, 21:20-23

19. Peng X, Kraus MS, Wei H, Shen TL, Pariaut R, Alcaraz A, Ji G, Cheng L, Yang Q, Kotlikoff MI, Chen J, Chien K, Gu H, Guan JL: Inactivation of focal adhesion kinase in cardiomyocytes promotes eccentric cardiac hypertrophy and fibrosis in mice. J Clin Invest 2006, 116: 217-227

20. Clement S, Stouffs M, Bettiol E, Kampf S, Krause KH, Chaponnier C, Jaconi M: Expression and function of alpha-smooth muscle actin during embryonic-stem-cell-derived cardiomyocyte differentiation. J Cell Sci 2007, 120:229-238

21. Papp S, Szabo E, Kim H, McCulloch CA, Opas M: Kinase-dependent adhesion to fibronectin: regulation by calreticulin. Exp Cell Res 2008, 314:1313-1326

22. Papp S, Fadel MP, Kim H, McCulloch CA, Opas M: Calreticulin affects fibronectin-based cell-substratum adhesion via the regulation of c-src activity. J Biol Chem 2007, 282:16585-16598

23. Sicheri F, Kuriyan J: Structures of Src-family tyrosine kinases. Curr Opin Struct Biol 1997, 7:777-785

24. Shu L, Shayman JA: Src kinase mediates the regulation of phospholipase C-gamma activity by glycosphingolipids. J Biol Chem 2003, 278:31419-31425

25. Heineke J, Molkentin JD: Regulation of cardiac hypertrophy by intracellular signalling pathways. Nat Rev Mol Cell Biol 2006, 7:589-600

26. Mesaeli N, Nakamura K, Zvaritch E, Dickie P, Dziak E, Krause KH, Opas M, MacLennan DH, Michalak M: Calreticulin is essential for cardiac development. J Cell Biol 1999, 144:857-868

27. Michalak M, Groenendyk J, Szabo E, Gold LI, Opas M: Calreticulin, a multi-process calcium-buffering chaperone of the endoplasmic reticulum. Biochem J 2009, 417:651-666

28. Sugden PH: An overview of endothelin signaling in the cardiac myocyte. J Mol Cell Cardiol 2003, 35:871-886

29. Simonson MS, Wang Y, Herman WH: Nuclear signaling by endothelin-1 requires Src protein-tyrosine kinases. J Biol Chem 1996, 271:77-82

30. Herman WH, Simonson MS: Nuclear signaling by endothelin-1: a Ras pathway for activation of the c-fos serum response element. J Biol Chem 1995, 270:11654-11661

31. Deng XF, Rokosh DG, Simpson PC: Autonomous and growth factorinduced hypertrophy in cultured neonatal mouse cardiac myocytes: comparison with rat. Circ Res 2000, 87:781-788

32. Karliner JS, Honbo N, Epstein CJ, Xian M, Lau YF, Gray MO: Neonatal mouse cardiac myocytes exhibit cardioprotection induced by hypoxic and pharmacologic preconditioning and by transgenic overexpression of human Cu/Zn superoxide dismutase. J Mol Cell Cardiol 2000, 32:1779-1786 